\title{
Colorful invasion in permissive Neotropical ecosystems: establishment of ornamental non-native poeciliids of the genera Poecilia/Xiphophorus (Cyprinodontiformes: Poeciliidae) and management alternatives
}

\author{
André Lincoln Barroso Magalhães ${ }^{1}$ and Claudia Maria Jacobi ${ }^{2}$
}

Headwater creeks are environments susceptible to invasion by non-native fishes. We evaluated the reproduction of 22 populations of the non-native livebearers guppy Poecilia reticulata, black molly Poecilia sphenops, Yucatan molly Poecilia velifera, green swordtail Xiphophorus hellerii, southern platyfish Xiphophorus maculatus, and variable platyfish Xiphophorus variatus during an annual cycle in five headwater creeks located in the largest South American ornamental aquaculture center, Paraíba do Sul River basin, southeastern Brazil. With few exceptions, females of most species were found reproducing (stages $2,3,4)$ all year round in the creeks and gravid females of all species showed small sizes indicating stunting. Juveniles were frequent in all sites. The fecundity of the six poeciliids was always low in all periods. The sex ratio was biased for females in most species, both bimonthly as for the whole period. Water temperature, water level and rainfall were not significantly correlated with reproduction in any species. Therefore, most populations appeared well established. The pertinence of different management actions, such as devices to prevent fish escape, eradication with rotenone and research about negative effects on native species, is discussed in the light of current aquaculture practices in the region.

Keywords: Aquaculture, Invasive species, Livebearers, Reproduction, Stream.

Riachos de cabeceira são ambientes susceptíveis à invasão por peixes não-nativos. Neste trabalho, avaliou-se a reprodução de 22 populações dos poecilídeos não-nativos guppy Poecilia reticulata, molinésia preta Poecilia sphenops, molinésia Poecilia velifera, espadinha Xiphophorus hellerii, plati Xiphophorus maculatus e plati variado Xiphophorus variatus durante diferentes anos em cinco riachos de cabeceira localizados no maior polo de piscicultura ornamental da América do Sul, bacia do rio Paraíba do Sul, sudeste do Brasil. Foram encontradas fêmeas da maioria das espécies em reprodução (estágios 2, 3, 4), durante todos os anos nos riachos e fêmeas grávidas de todas as espécies apresentaram pequeno tamanho indicando nanismo. Juvenis de todas as espécies foram frequentes em todos os locais. A fecundidade das seis espécies sempre foi baixa em todos os períodos. Para os períodos bimestrais e totais, encontrou-se mais fêmeas que machos na maioria das espécies. Temperatura e nível de água dos riachos, bem como precipitação pluviométrica não foram correlacionadas com a reprodução. Todas as populações estão estabelecidas nos locais e ações de gerenciamento como dispositivos para impedir a fuga de peixes, erradicação com rotenona e pesquisa científica sobre os efeitos negativos nas espécies nativas são discutidas em relação às práticas de piscicultura na região.

Palavras-chave: Aquicultura, Córrego, Espécies invasoras, Ovovivíparos, Reprodução.

\section{Introduction}

Headwater creeks occur in all landscapes around the globe, and may compose almost $80 \%$ of total stream length in many drainage networks (Sidle et al., 2000). They offer an enormous array of habitats for microbial, plant, and animal life, and are sensitive areas for the conservation of aquatic biodiversity due to their low species richness and high degree of endemism (Abell et al., 2007). These characteristics define them as permissive environments, i.e. susceptible to the establishment of non-native species (Courtenay et al., 1974), and are among the most threatened systems in the planet (Dudgeon et al., 2006).

In South America and Brazil, native communities in headwater creeks are suffering changes in their original structure due to changes in riparian vegetation, siltation, pollution, small dams, recreational activities, cattle ranching and non-native fish introductions resulting from human involvement (Bizerril, Lima, 2001; Castellani, Barrella, 2006; Casatti et al., 2009; Magalhães, Jacobi, 2013a).

${ }^{1}$ Programa de Pós-graduação em Tecnologias para o Desenvolvimento Sustentável, Universidade Federal de São João Del Rei, Rodovia MG 443, KM 7, Fazenda do Cadete, 36420-000 Ouro Branco, MG, Brazil. andrebiomagalhaes@gmail.com (corresponding author) ${ }^{2}$ Departamento de Biologia Geral, Universidade Federal de Minas Gerais, Avenida Antônio Carlos, 6227, 31270-901 Belo Horizonte, MG, Brazil.jacobi@icb.ufmg.br 
Although more than 50 non-native fish species are currently found in Brazilian headwater creeks (Bizerril, Lima, 2001; Magalhães, Jacobi, 2008), a group of seven poeciliids deserve special attention as a threat to native communities due to negative impacts such as changes in the structure of the native fish assemblages and biotic homogenization: the guppy Poecilia reticulata Peters, 1859, sailfin molly P. latipinna (Lesueur 1821), black molly P. sphenops Valenciennes in Cuvier, Valenciennes, 1846, Yucatan molly $P$. velifera (Regan 1914), green swordtail Xiphophorus hellerii Heckel 1848, southern platyfish $X$. maculatus (Günther 1866), and variable platyfish $X$. variatus (Meek 1904) (Alves et al., 2007; Magalhães, Jacobi, 2008; Magalhães et al., 2011). Along with cyprinids, cichlids and osphronemids, these poeciliids are the ornamental fishes most sought by the Brazilian aquarium trade (Coe et al., 2011; Magalhães, Jacobi, 2013b; Assis et al., 2014; Garcia et al., 2014). They all have been introduced into headwater creeks of the southeastern region mainly as by-products of aquaculture-related activities (Bizerril, Lima, 2001; Castellani, Barrella, 2006; Alves et al., 2007; Magalhães, Jacobi, 2008). Poeciliidae, however, has been identified as one of the top invasive freshwater fish families and is linked to reduction in zooplankton, damselfly/dragonfly populations, decline and extinction of native fishes and also to negatively impact native amphibians by preying on amphibian eggs and larval stages in several regions of the world (Stockwell, Henkanaththegedara, 2011). Because they are livebearers, their members have an invasive advantage over species of other popular non-native families (Milton, Arthington, 1983; Deacon et al., 2011).

It is the initial and final stages of biological invasions that are usually the target of most studies in this field, but intermediate stages, involving the dynamics of reproduction, should deserve equal attention (Williamson, 2000). Reproductive characteristics have substantial effects on a species invasion success, and a thorough knowledge of the reproductive biology of poeciliids outside their natural ranges is a fundamental prerequisite to assess the extent of their establishment and potential ecological impacts on native communities of headwater creeks.

Thus, our goal was to evaluate the reproductive status (i.e. establishment) of 22 populations of non-native aquarium poeciliids in five headwater Brazilian creeks, determine their response to local abiotic factors (water temperature/level, rainfall), discuss their impacts and suggest management measures to protect these peculiar ecosystems.

\section{Material and Methods}

Study area. The Muriaé Ornamental Aquaculture Center (MOAC) was founded in 1979 at southeast of Minas Gerais State, Brazil. MOAC is the largest ornamental aquaculture area in South America, comprising 13 municipalities, two villages and is currently home to 350 fish farms, 4,500 fish ponds (Magalhães, Jacobi, 2008). This area provides $70 \%$ of the Brazilian aquarium fishes demand (Cardoso, Igarashi, 2009). The poeciliids $P$. reticulata, $P$. sphenops, $X$. hellerii and $X$. maculatus are among the 20 most cultured species, with a combined annual production of almost 3,000,000 individuals raised in monoculture ponds (Cardoso et al., 2012).

Field collections. The vegetation cover in the region consists of Atlantic Forest remnants (i.e. mesophyllous semideciduous forest) interspersed with grazing and monoculture cultivations initiated since the 1940's. Five headwater creeks (first-order, sensu Strahler, 1957) were surveyed, all belonging to the Paraíba do Sul River basin, one of the main South American watersheds (Albert, Reis, 2011). These creeks run across a region with hundreds of culture ponds surrounding the major municipalities of the aquaculture center. They are slow-flowing warmwater habitats, have clean water; with an average width of $137 \mathrm{~cm}$, sandy/muddy bottoms devoid of aquatic macrophytes, their mud banks do not have riparian vegetation, are covered by non-native grasses Brachiaria spp. (syn. Urochloa spp.) due to cattle ranching, and are subjected to flash floods in the rainy season (Magalhães, Jacobi, 2013a). Their native ichthyofauna is composed of a few small characids, cichlids, loricariids, trichomycterids, and poeciliids like the spotsided livebearer Poecilia vivipara Bloch \& Schneider 1801 and the endemic dusky millions fish Phalloceros cf. leptokeras Lucinda 2008 (Vieira, Rodrigues, 2010). In all sites of this study, the 369 production ponds are drained every 45 days, which represents almost 3,000 pond drainage events per year. This practice creates a massive propagule pressure, given that ponds have no screens on the effluent pipes to prevent escapes.

Fish were collected every two months, from January to December, in different years in each creek: Boa Vista

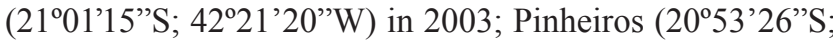
$\left.42^{\circ} 20^{\prime} 33^{\prime \prime} \mathrm{W}\right)$ in 2004; Santo Antônio (20'53'14"S; $\left.42^{\circ} 17^{\prime} 26^{\prime \prime} \mathrm{W}\right)$ in 2005; Chato (2053'28'S; 42'22'36'W) and Gavião (2054'30"S; 42 $24^{\prime} 38^{\prime \prime} \mathrm{W}$ ) in 2006, southeastern Brazil (Fig. 1). Juveniles and adults were collected by the same person from margins and channel beds, with a rectangular sieve measuring $95 \mathrm{~cm}$ long $\times 25 \mathrm{~cm}$ high and $0.3 \mathrm{~mm}$ mesh. The sieve was cast 50 times along 100 $\mathrm{m}$-long transects during $2 \mathrm{~h}$ in each creek at daytime. The fishes (Tab. 1) were euthanized on an ice slurry, a method approved for field work by IACUC (2002), sorted by site collection, packed in plastic bags, fixed in 10\% formalin, and subsequently transferred to $70 \%$ alcohol. Voucher specimens used in this study are deposited under their respective catalogue numbers in the Museu de Ciências e Tecnologia da Pontifícia Universidade Católica do Rio Grande do Sul (MCP) and in the Universidade Federal de Sergipe fish collection (CIUFS). In the laboratory, the species were measured (standard length SL - given in $\mathrm{cm}$ ) and sexed as males if there was any evidence of a gonopodium, as juveniles if smaller than the smallest male in each sample, and as females in the absence of a gonopodium but larger than the smallest male (Gkenas et al., 2012). 


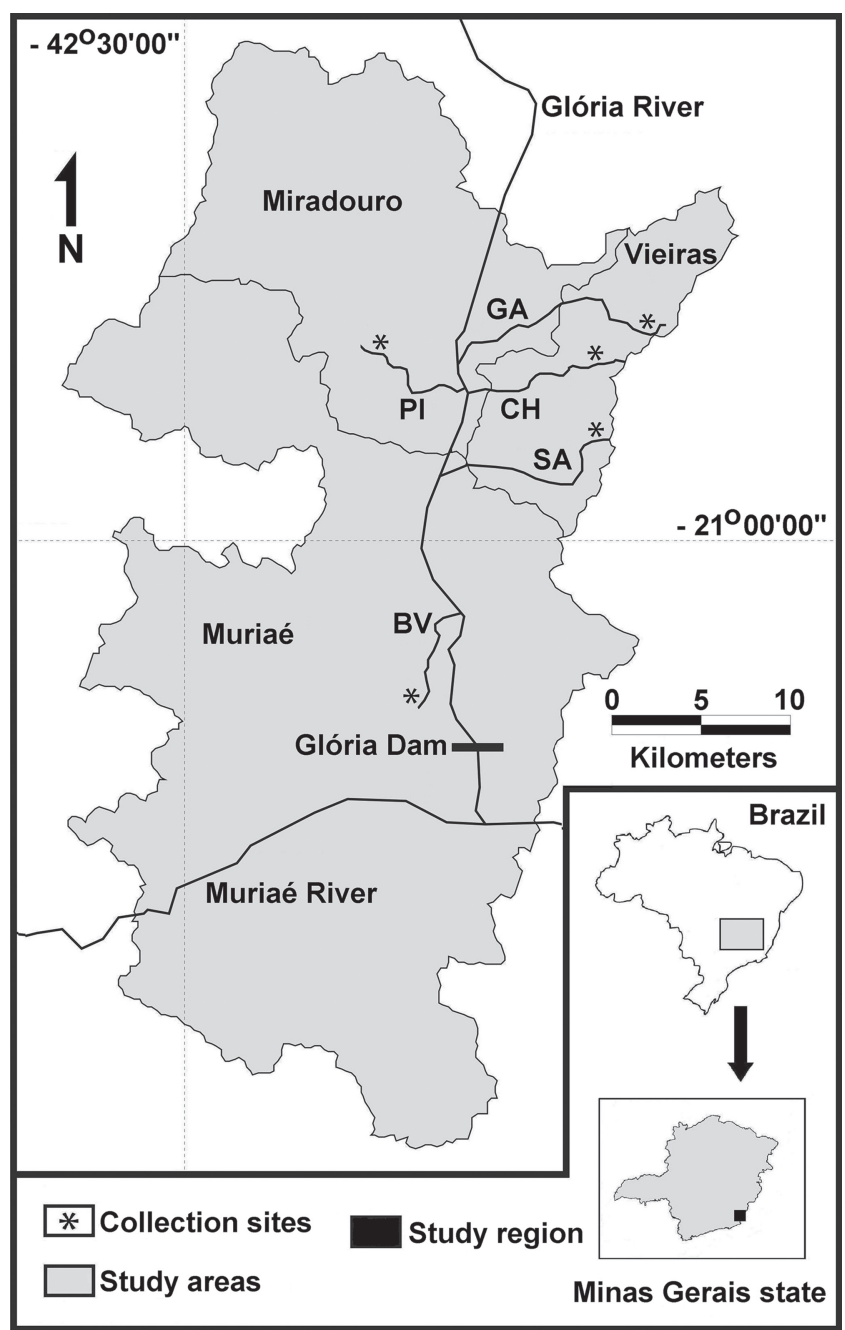

Fig. 1. Sampling locations of Poecilia and Xiphophorus species. Municipalities: Muriaé, Miradouro, Vieiras. Headwater creeks: BV Boa Vista, PI Pinheiros, SA Santo Antônio, CH Chato, GA Gavião (modified from Magalhães, Jacobi, 2013a).
Developmental stages. We adapted the methods of Winemiller (1993) (macroscopic) and Haynes (1995) (mesoscopic) to determine the developmental stages of adult females. We classified as non-gravid females those without a conspicuously enlarged ventral region, absence of eggs, embryos, or gravid spot near the base of the anal fin. On the other hand, females with an enlarged belly, presence of gravid spot (not seen in females of $P$. sphenops and $P$. velifera due to their solid black and marble colours respectively), eggs, or embryos seen with the naked eye were classified as gravid females. On a mesoscopic scale, developmental stages were classified as follows: Non-gravid (stage 1 - ovarian tissue without ovum and/or embryos), Yolked ova (stage 2 - ovum in the process of yolking, yellow in guppy, green swordtail, southern platyfish, variable platyfish and orange in black molly, Yucatan molly; oil droplets, if present, unevenly distributed), Eyed embryos (stage 3 - eyes pigmented, head enlarged compared to trunk, caudal and pectoral fin buds present, dorsal pigmentation initiated in all species), Mature embryos (stage 4 - yolk sac mostly or completely absorbed, pectoral fins elongate, scales present, embryo resembling a small adult). After determining the developmental stages, the bimonthly absolute and relative frequencies of females were calculated.

Population structure, fecundity and sex ratio. The adult-tojuvenile absolute and relative frequencies and ratio (Pope et al., 2010) were calculated from the total number of females, males and juveniles of all poeciliid species. We estimated the bimonthly and total mean values of fecundity (embryos in stages 3 and 4 ) of all species in the creeks. Only embryos were used as a measure of fecundity because in the ova development is not readily discernible with a dissecting microscope and it is not known whether they have been fertilized (Haynes, 1995). The sex ratio (Pope et al., 2010) was calculated from the bimonthly and total number of females and males of the six species collected in the five creeks.

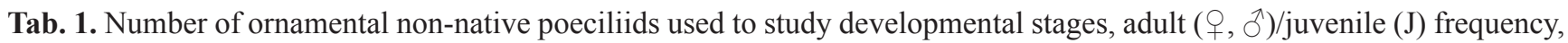
fecundity and sex ratios in 2003, 2004, 2005 and 2006.

\begin{tabular}{|c|c|c|c|c|c|c|c|c|c|c|c|c|c|c|c|}
\hline \multirow{2}{*}{$\begin{array}{c}\text { Species } \\
\text { Voucher specimens }\end{array}$} & \multicolumn{3}{|c|}{ Boa Vista } & \multicolumn{3}{|c|}{ Pinheiros } & \multicolumn{3}{|c|}{ Santo Antônio } & \multicolumn{3}{|c|}{ Chato } & \multicolumn{3}{|c|}{ Gavião } \\
\hline & q & $\hat{\sigma}$ & $\mathrm{J}$ & q & $\hat{\sigma}$ & $\mathrm{J}$ & 우 & 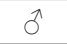 & $\mathrm{J}$ & q & o & $\mathrm{J}$ & 우 & $\hat{\sigma}$ & $\mathrm{J}$ \\
\hline Poecilia reticulata & 87 & 24 & 442 & 103 & 104 & 531 & 514 & 192 & 2,258 & 601 & 401 & 3,427 & 590 & 317 & 1,270 \\
\hline Voucher number & \multicolumn{3}{|c|}{ CIUFS 2601} & \multicolumn{3}{|c|}{ CIUFS 2604} & \multicolumn{3}{|c|}{ CIUFS 2597} & \multicolumn{3}{|c|}{ CIUFS 2608} & \multicolumn{3}{|c|}{ CIUFS 2613} \\
\hline Poecilia sphenops & \multicolumn{3}{|c|}{-} & \multicolumn{3}{|c|}{-} & 168 & 91 & 341 & 242 & 143 & 175 & \multicolumn{3}{|c|}{-} \\
\hline Voucher number & \multicolumn{3}{|c|}{-} & \multicolumn{3}{|c|}{-} & \multicolumn{3}{|c|}{ CIUFS 2600} & \multicolumn{3}{|c|}{ CIUFS 2611} & & - & \\
\hline Poecilia velifera & & - & & & - & & 97 & 53 & 243 & \multicolumn{3}{|c|}{-} & \multicolumn{3}{|c|}{-} \\
\hline Voucher number & \multicolumn{3}{|c|}{-} & & - & & \multicolumn{3}{|c|}{ MCP 39003} & \multicolumn{3}{|c|}{-} & \multicolumn{3}{|c|}{ - } \\
\hline Xiphophorus hellerii & 140 & 56 & 203 & 145 & 43 & 225 & 208 & 108 & 401 & 363 & 195 & 697 & 536 & 76 & 530 \\
\hline Voucher number & \multicolumn{3}{|c|}{ CIUFS 2603} & \multicolumn{3}{|c|}{ CIUFS 2606} & \multicolumn{3}{|c|}{ CIUFS 2599} & \multicolumn{3}{|c|}{ CIUFS 2610} & \multicolumn{3}{|c|}{ CIUFS 2614} \\
\hline Xiphophorus maculatus & 80 & 77 & 151 & 161 & 84 & 138 & 128 & 132 & 110 & 261 & 156 & 120 & 355 & 207 & 133 \\
\hline Voucher number & \multicolumn{3}{|c|}{ CIUFS 2602} & \multicolumn{3}{|c|}{ CIUFS 2605} & \multicolumn{3}{|c|}{ CIUFS 2598} & \multicolumn{3}{|c|}{ CIUFS 2609} & \multicolumn{3}{|c|}{ CIUFS 2615} \\
\hline Xiphophorus variatus & & - & & 119 & 103 & 351 & 278 & 75 & 269 & 284 & 104 & 494 & 538 & 265 & 1,002 \\
\hline Voucher number & & - & & & JFS 2 & & & CP 390 & & & JFS 2 & & & JFS 2 & \\
\hline
\end{tabular}


Correlation between reproduction and abiotic factors. Abiotic data were collected every two months, namely water temperature, level, and rainfall. The first two were obtained at the times of the fish sampling in each creek. We measured water temperature in the field with a mercury-in-glass thermometer to the scale $0^{\circ}$ to $50^{\circ} \mathrm{C}$, and water level using a pole marked every $10 \mathrm{~cm}$. Rainfall data are from the database of the INPE (2006) located in the municipalities of Muriaé, Miradouro, and Vieiras. Following Andrade, Braga (2005), we grouped the bimonthly relative frequencies of gravid females (stages 2, 3, and 4), which were then correlated with the average bimonthly water temperature, water level and rainfall.

Statistical analyses. Chi-square $\left(\chi^{2}\right.$ test) goodness of fit tests were conducted to determine the differences in proportions between juveniles-to-adults and sexes, and the Spearman's rank correlation to test a relationship between female reproduction and abiotic factors (Sokal, Rohlf, 1995). Differences were significant when $\mathrm{P}<$ 0.05. All statistical analyses were performed using PAST-Paleontological Statistics (version 1.91) software (Hammer et al., 2009).

\section{Results}

Developmental stages and early maturity. All poeciliid species showed a long reproductive period, varying from eight to 12 months (Figs. 2-6). Females were found in several developmental stages during almost all the sampling period in the creeks. The exceptions were $P$. reticulata in Boa Vista Creek where females showed no reproductive activity in March/April 2003 (Fig. 2), and $P$. velifera in Santo Antônio Creek, which were not collected from March to June 2005 (Fig. 4). Although all species showed reproductive activity, the proportion of non-gravid females differed among species. In particular, $P$. sphenops, $P$. velifera, and $X$. hellerii showed low percentages of gravid females in most bimesters, while $X$ variatus was rarely under $40 \%$. These proportions also differed among creeks, with Santo Antônio showing a higher reproductive activity (Fig. 4), and Gavião with less (Fig. 6). In Boa Vista Creek, the smallest gravid female of $P$. reticulata measured $1.8 \mathrm{~cm} \mathrm{SL}, X$. hellerii $3.3 \mathrm{~cm}$ SL and $X$. maculatus measured $2.8 \mathrm{~cm}$ SL. In Pinheiros Creek, $P$. reticulata measured $1.9 \mathrm{~cm}$ SL, $X$. hellerii $3.5 \mathrm{~cm} \mathrm{SL}, X$. maculatus $2.8 \mathrm{~cm} \mathrm{SL}$ and $X$. variatus measured $2.9 \mathrm{~cm}$ SL. In Santo Antônio Creek, $P$. reticulata $2.1 \mathrm{~cm} \mathrm{SL}, P$. sphenops $3.0 \mathrm{~cm}, P$. velifera $3.0 \mathrm{~cm} \mathrm{SL}, X$. hellerii $3.2 \mathrm{~cm}, X$. maculatus $2.6 \mathrm{~cm} \mathrm{SL}$ and $X$. variatus measured $2.7 \mathrm{~cm}$ SL. In Chato Creek, $P$. reticulata female measured $2.1 \mathrm{~cm}$ SL, $P$. sphenops $2.5 \mathrm{~cm} \mathrm{SL}, X$. hellerii $3.2 \mathrm{~cm}$ SL, $X$. maculatus $2.5 \mathrm{~cm}$ $\mathrm{SL}, X$. variatus $2.4 \mathrm{~cm} \mathrm{SL}$, and in the Gavião Creek, the smallest gravid female of $P$. reticulata measured $2.0 \mathrm{~cm}$ $\mathrm{SL}, X$. hellerii $2.5 \mathrm{~cm} \mathrm{SL}, X$. maculatus $1.9 \mathrm{~cm} \mathrm{SL}$ and $X$. variatus measured $2.2 \mathrm{~cm} \mathrm{SL}$.
Poecilia reticulata

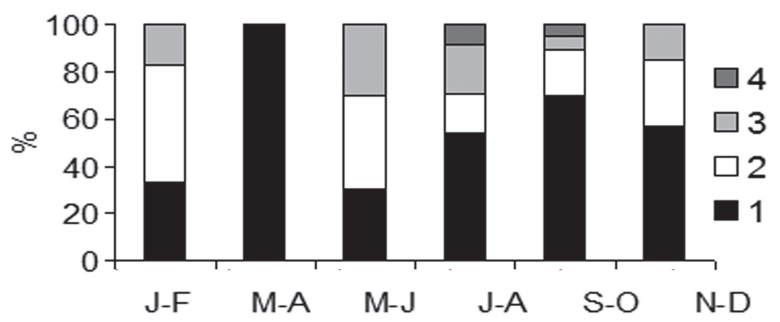

Xiphophorus hellerii

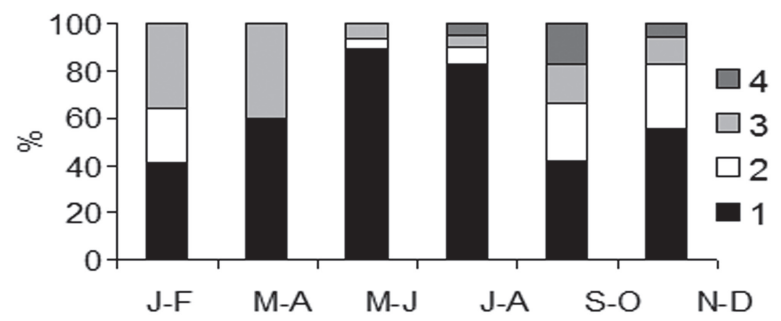

Xiphophorus maculatus

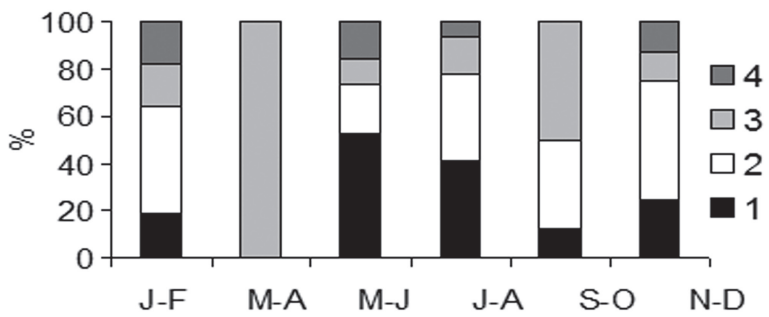

Fig. 2. Relative bimonthly frequency of developmental stages in ornamental non-native poeciliid females collected in Boa Vista Creek, 2003. Developmental stages: 1 Non-gravid, 2 Yolked ova, 3 Eyed embryos, 4 Mature embryos. 


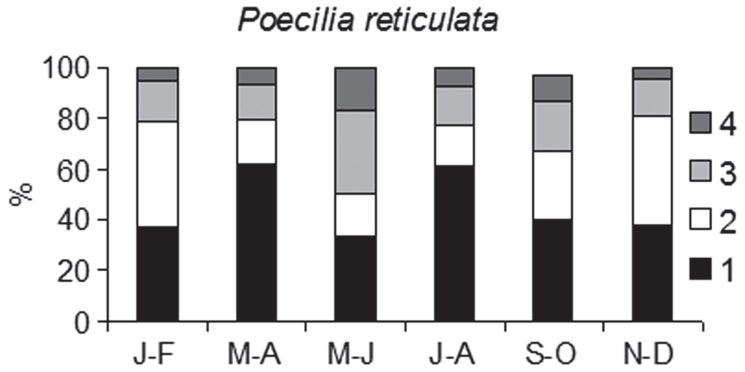

Xiphophorus maculatus

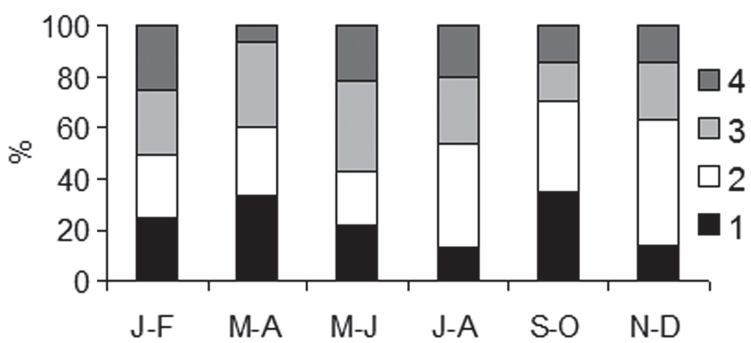

Xiphophorus hellerii

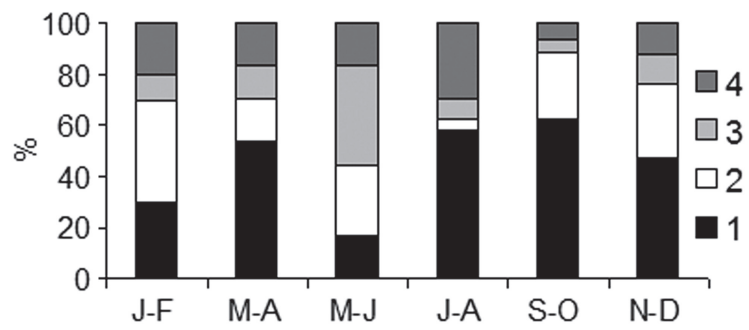

Xiphophorus variatus

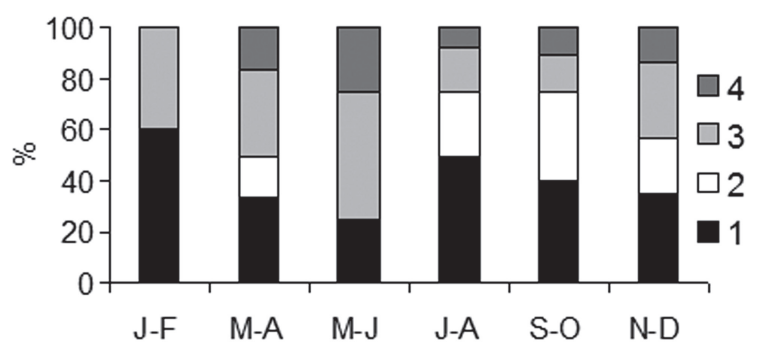

Fig. 3. Relative bimonthly frequency of developmental stages in ornamental non-native poeciliid females collected in Pinheiros Creek, 2004. Developmental stages: 1 Non-gravid, 2 Yolked ova, 3 Eyed embryos, 4 Mature embryos.
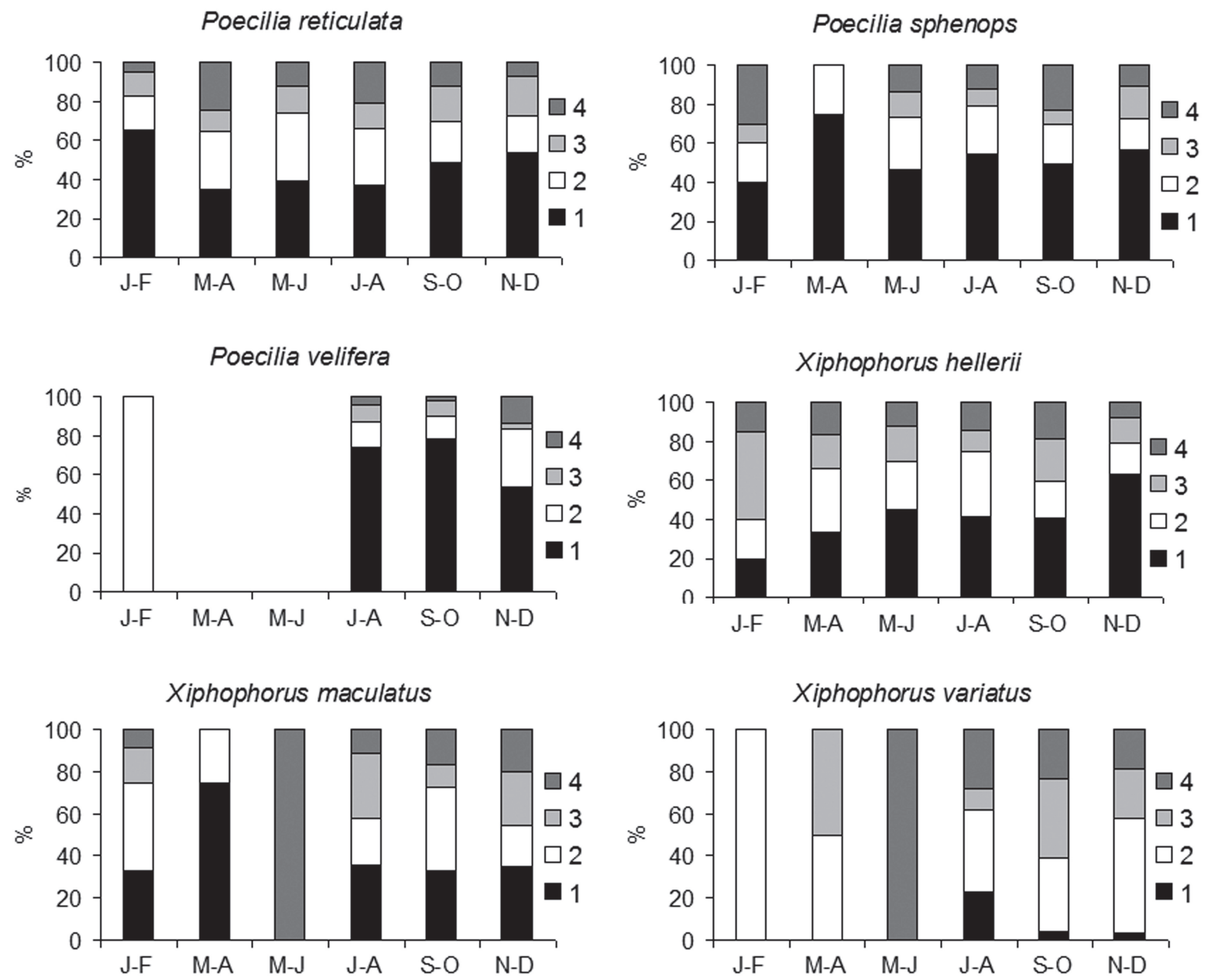

Fig. 4. Relative bimonthly frequency of developmental stages in ornamental non-native poeciliid females collected in Santo Antônio Creek, 2005. Developmental stages: 1 Non-gravid, 2 Yolked ova, 3 Eyed embryos, 4 Mature embryos. 

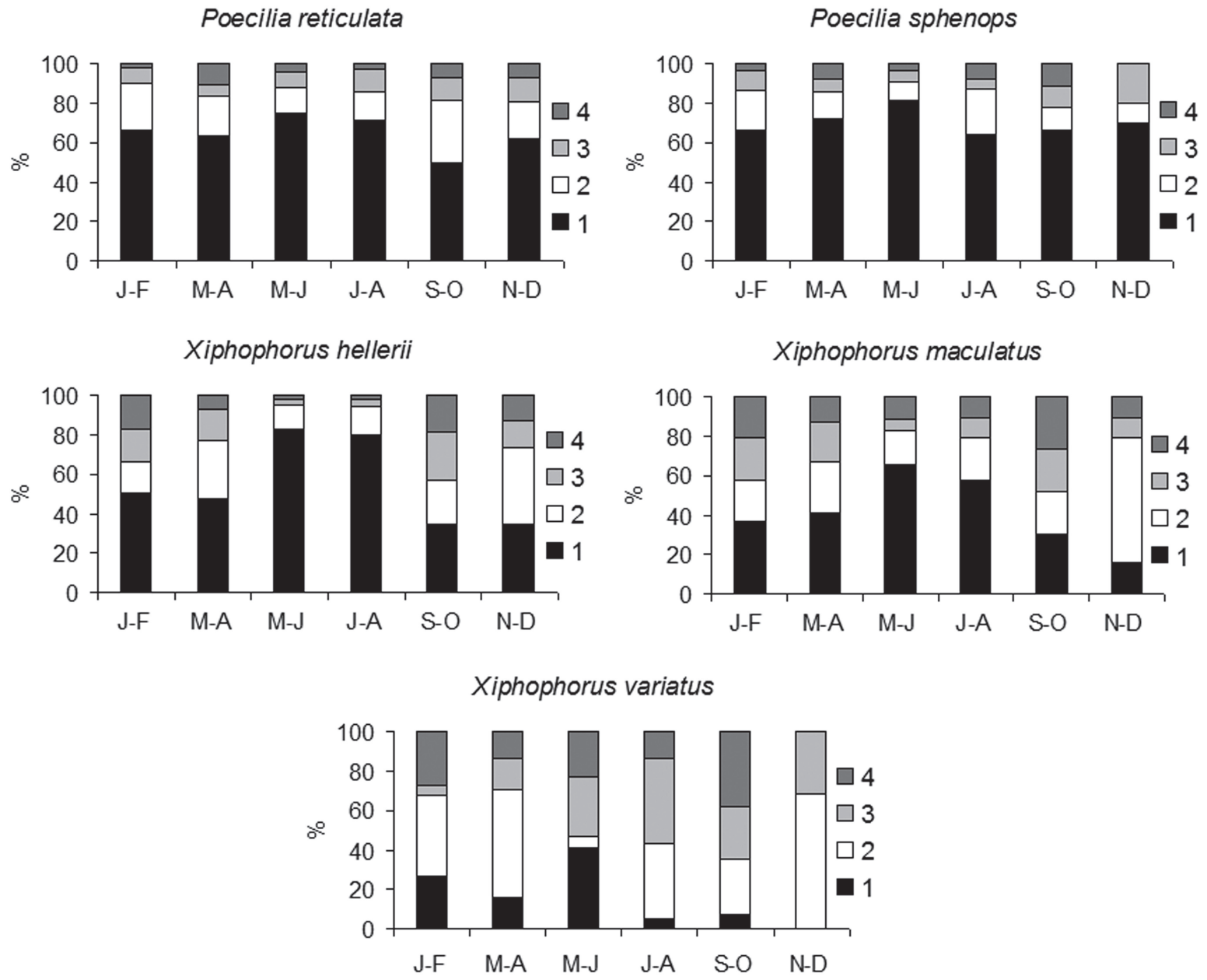

Fig. 5. Relative bimonthly frequency of developmental stages in ornamental non-native poeciliid females collected in Chato Creek, 2006. Developmental stages: 1 Non-gravid, 2 Yolked ova, 3 Eyed embryos, 4 Mature embryos.
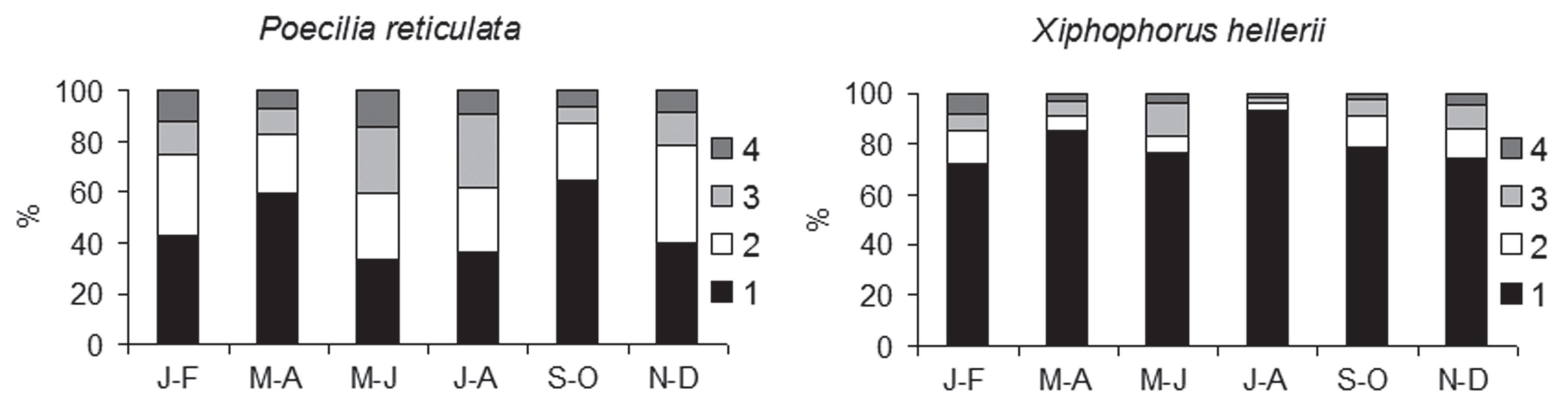

Xiphophorus maculatus

Xiphophorus variatus
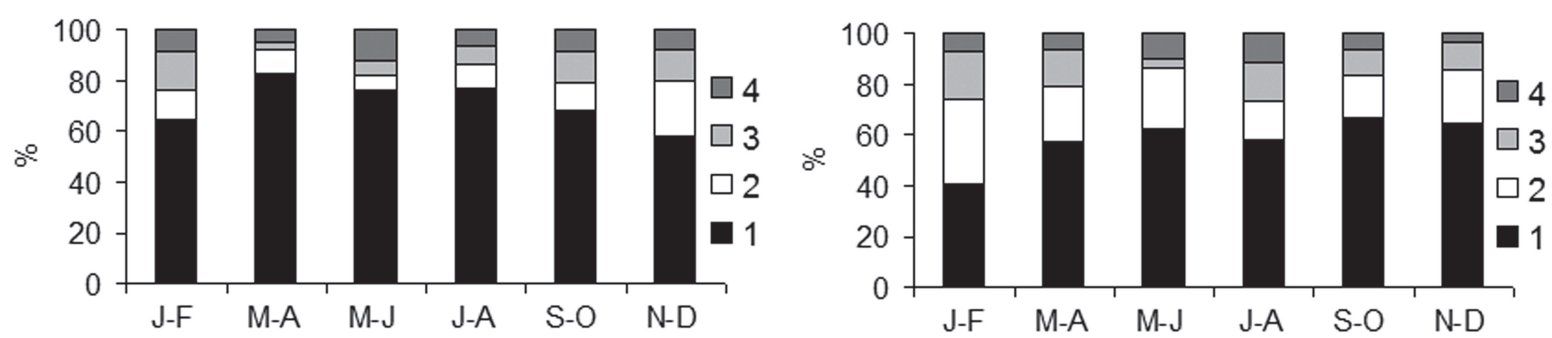

Fig. 6. Relative bimonthly frequency of developmental stages in ornamental non-native poeciliid females collected in Gavião Creek, 2006. Developmental stages: 1 Non-gravid, 2 Yolked ova, 3 Eyed embryos, 4 Mature embryos. 
Population structure, fecundity and sex ratio. Nonnative juvenile poeciliids of undetermined sex were caught in all sites. Non-native juveniles had a total number of fish captured of 13,511 specimens and a proportion of $95.17 \%$. These values represent significant amounts and proportion when compared with the low number (686) and proportion of native juveniles $(4.83 \%)$. For the total period, the number of non-native juvenile poeciliids was higher than adult females and males in 12 populations (54.54\%). Conversely, the number of juveniles was lower than adult females and males in seven populations $(31.82 \%)$ captured in Pinheiros ( $X$. maculatus $63.97 \% ; \chi^{2}=29.9$; d.f. $=1 ; \mathrm{P}<0.05$ ), Santo Antônio (X. maculatus 70,27\%; $\chi^{2}=60.8$; d.f. $=1 ; \mathrm{P}<0.05, X$. variatus $56.75 \% ; \chi^{2}=11.34$; d.f. $=1 ; \mathrm{P}<0.05$ ), Chato ( $P$. sphenops $68.75 \% ; \chi^{2}=78.75$; d.f. $=1 ; \mathrm{P}<0.05, X$. maculatus $77.65 \% ; \chi^{2}=164.26$; d.f. $=1$; $\mathrm{P}<0.05)$ and Gavião $\left(X\right.$. hellerii $53.59 \% ; \chi^{2}=5.88$; d.f. $=1$; $\mathrm{P}<0.05$, X. maculatus $80.86 \%$; $\chi^{2}=264.8$; d.f. $=1 ; \mathrm{P}<0.05$ ) creeks (Tab. 2).

The mean fecundity of the six species was low in all bimesters and also for the total period in comparison with other introduced populations. The lowest fecundities, below seven embryos per brood, were observed in $P$. reticulata in all creeks $(4.81 \pm 0.62,6.05 \pm 1.59,5.93 \pm 0.90$, $5.25 \pm 1.19,6.14 \pm 1.61)$, X. maculatus in Santo Antônio and Chato creeks respectively $(6.59 \pm 1.98,6.23 \pm 0.44)$, and $X$. variatus in Gavião Creek (6.72 \pm 1.60$)$. The highest values corresponded to $X$. helleri in Pinheiros Creek, with more than 10 embryos per brood (Fig. 7).
Overall, females were significantly more abundant than males in all sites. In Boa Vista Creek, females were more frequent than males in guppy $\left(\chi^{2}=34.94 ; 3.5: 1\right.$; d.f. $=1 ; \mathrm{P}<0.05)$ and green swordtail $\left(\chi^{2}=36.00 ; 2.5: 1\right.$; d.f. $=1 ; \mathrm{P}<0.05)$. We found no significant differences in sex ratio of southern platyfish $\left(\chi^{2}=0.00 ; 1: 1\right.$; d.f. $=1$; $\mathrm{P}<0.05)$. In Pinheiros Creek, we found more females than males in $X$. hellerii $\left(\chi^{2}=55.34 ; 3.40: 1\right.$; d.f. $=1$; $\mathrm{P}<$ $0.05)$ and $X$. maculatus $\left(\chi^{2}=24.20 ; 1.90: 1 ;\right.$ d.f. $=1 ; \mathrm{P}<$ $0.05)$, whereas the sex ratio of $P$. reticulata and variable platyfish was almost identical $\left(\chi^{2}=0.00 ; 0.99: 1\right.$; d.f. $=1$; $\mathrm{P}<0.05, \chi^{2}=1.16 ; 1.15: 1 ;$ d.f. $\left.=1 ; \mathrm{P}<0.05\right)$. In Santo Antônio Creek, females were significantly more frequent than males in $P$. reticulata, $P$. sphenops, $P$. velifera, $X$. hellerii, and $X$. variatus. On the other hand, males and females had the same frequencies in $X$. maculatus $\left(\chi^{2}=0.06 ; 0.97: 1 ;\right.$ d.f. $\left.=1 ; \mathrm{P}<0.05\right)$. In Chato Creek, females were significantly more frequent than males in all five species. In Gavião Creek, females of $P$. reticulata, $X$. hellerii, $X$. maculatus, and $X$. variatus were significantly more frequent than males $\left(\chi^{2}=82.18 ; 1.86: 1\right.$; d.f. $=1$; P $<0.05, \chi^{2}=345.75 ; 7.05: 1$; d.f. $=1 ; \mathrm{P}<0.05, \chi^{2}=38.98$; $1.71: 1 ;$ d.f. $=1 ; \mathrm{P}<0.05, \chi^{2}=92.82 ; 2.03: 1 ;$ d.f. $=1 ; \mathrm{P}<$ $0.05)$ (Tab. 3).

Correlation between reproduction and abiotic factors. Water temperature, water level, and rainfall were not significantly correlated with reproduction of females in any of the six species or creeks (Tab. 4).

Tab. 2. Total absolute/relative frequencies and adult-to-juvenile ratio of ornamental non-native poeciliid species collected in the creeks in 2003, 2004, 2005 and 2006. Adult $\left(q+{ }^{-}\right)$, juvenile $(\mathrm{J}) .{ }^{*}$ Indicates the significant values for adult-to-juvenile ratio (Chi-square, $\mathrm{P}<0.05, \chi^{2}{ }_{0.05}=3.841$, d.f. $=1$ ).

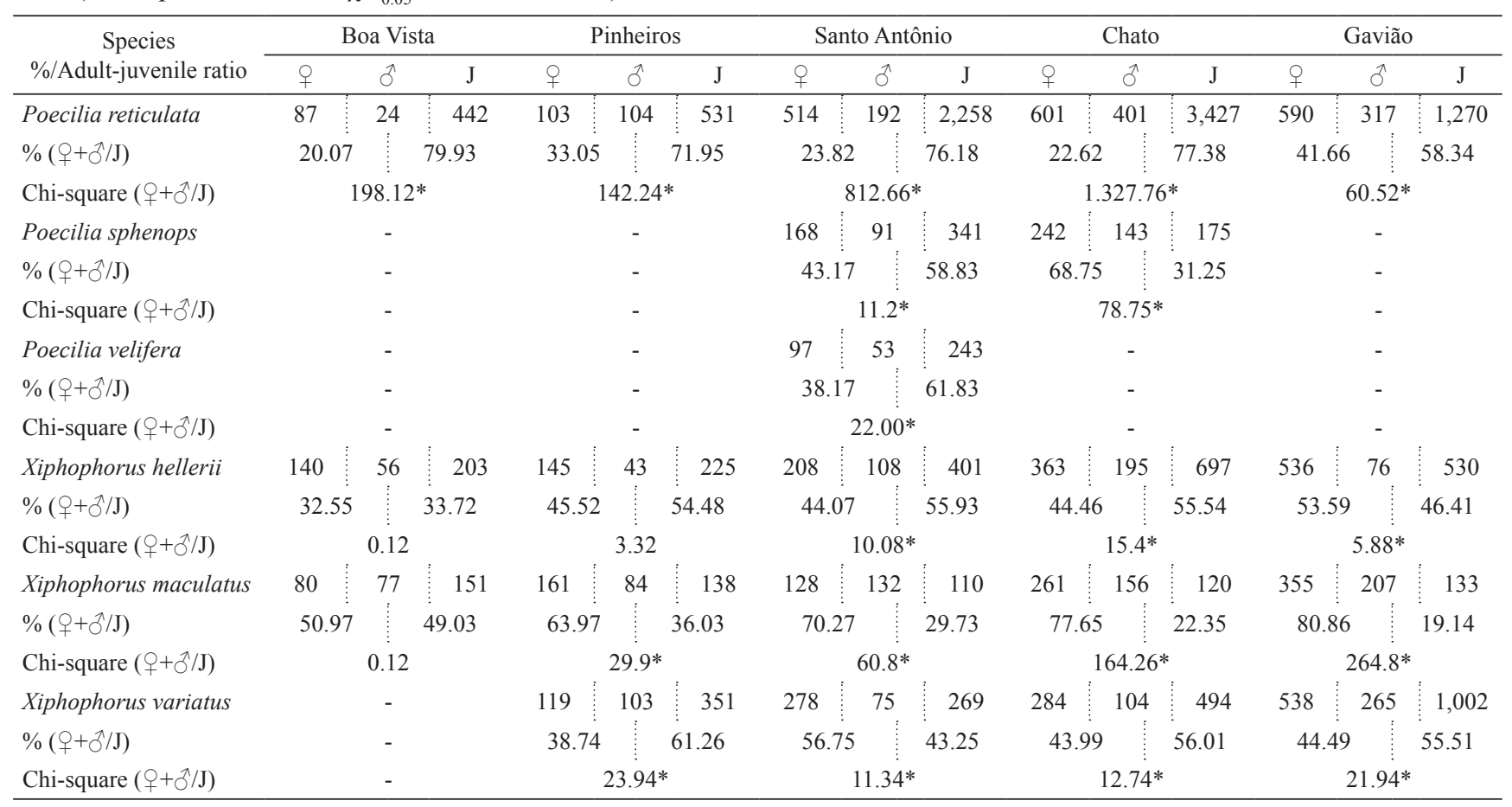



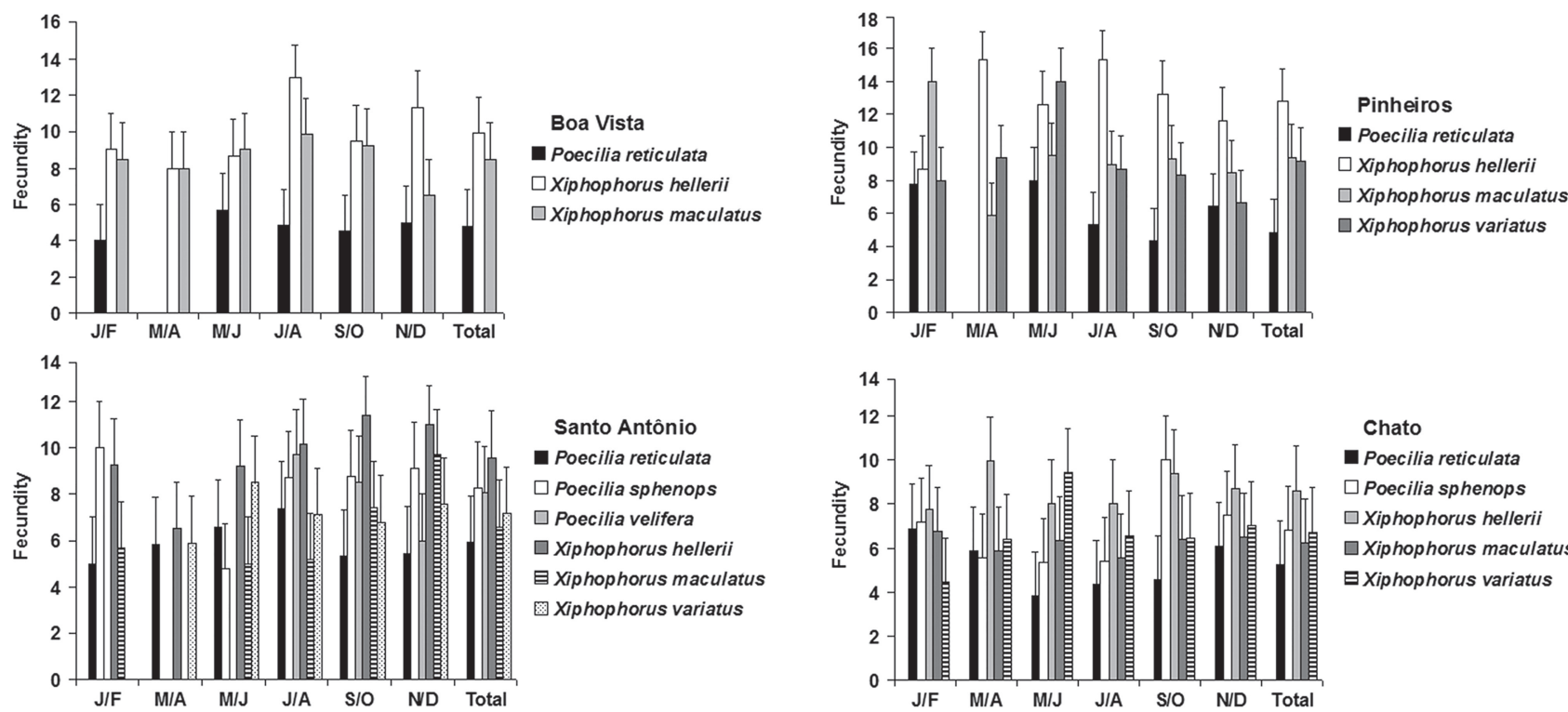

Santo Antônio

- Poecilia reticulata

$\square$ Poecilia sphenops

$\square$ Poecilia velifera

$\square$ Xiphophorus hellerii

目Xiphophorus maculatus

중 Xiphophorus variatus

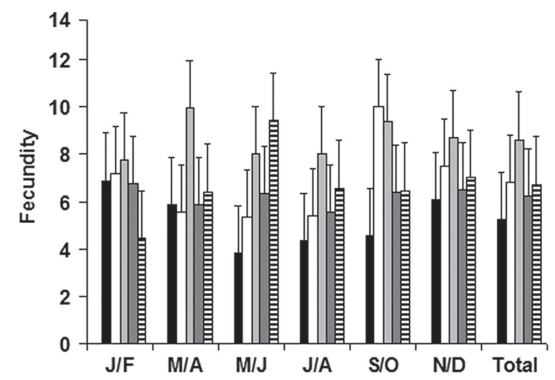

Chato

Poecilia reticulata

$\square$ Poecilia sphenops

$\square$ Xiphophorus hellerii

$\square$ Xiphophorus maculatus

EXiphophorus variatus

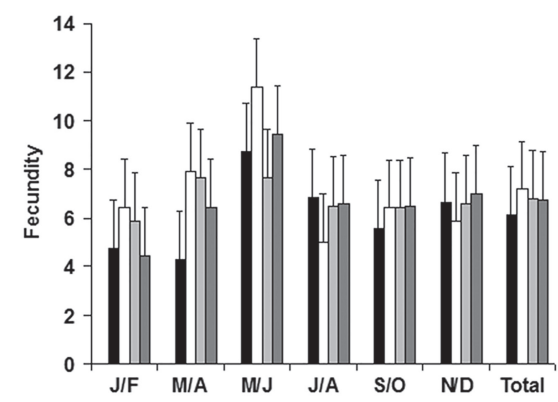

Gaviäo

Poecilia reticulata

$\square$ Xiphophorus hellerii

$\square$ Xiphophorus maculatus

$\square$ Xiphophorus variatus

Fig. 7. Bimonthly and total fecundity (number of embryos per brood) of ornamental non-native poeciliid species collected in the creeks in 2003, 2004, 2005 and 2006.

Tab. 3. Bimonthly and total sex ratio of ornamental non-native poeciliid species collected in the creeks in 2003, 2004, 2005 and 2006. * Indicates the significant values for sex ratio (Chi-square, $\mathrm{P}<0.05, \chi^{2}{ }_{0.05}=3.841$, d.f. $=1$ ).

\begin{tabular}{|c|c|c|c|c|c|}
\hline Species/Sex ratio & Boa Vista & Pinheiros & Santo Antônio & Chato & Gavião \\
\hline \multicolumn{6}{|l|}{ Poecilia reticulata } \\
\hline January-February & $5.40 *$ & 3.58 & $66.56^{*}$ & $5.36^{*}$ & $13.30^{*}$ \\
\hline March-April & 1.00 & 1.48 & $23.52 *$ & $4.26^{*}$ & $40.82 *$ \\
\hline May-June & $9.00 *$ & 0.00 & $13.12 *$ & $11.02 *$ & $12.48^{*}$ \\
\hline July-August & $9.32 *$ & 2.32 & $32.50 *$ & $4.78^{*}$ & $9.26^{*}$ \\
\hline September-October & $9.00 *$ & 0.14 & $12.94 *$ & $10.32 *$ & $4.94 *$ \\
\hline November-December & 1.60 & 1.00 & $16.82 *$ & $5.90^{*}$ & $11.74^{*}$ \\
\hline $\begin{array}{l}\text { Total/SR }+ \text { : } \hat{\jmath} \\
\quad \text { Poecilia sphenops }\end{array}$ & $34.94 * / 3.5$ & $0.00 / 0.99$ & $146.86 * / 2.68$ & $39.92 * / 1.50$ & $82.18 * / 1.86$ \\
\hline January-February & - & - & $7.36^{*}$ & $10.22 *$ & - \\
\hline March-April & - & - & 0.00 & $11.60^{*}$ & - \\
\hline May-June & - & - & $9.94 *$ & 1.14 & - \\
\hline July-August & - & - & $12.30^{*}$ & 3.58 & - \\
\hline September-October & - & - & $19.46^{*}$ & 1.14 & - \\
\hline November-December & - & - & 1.42 & 0.06 & - \\
\hline 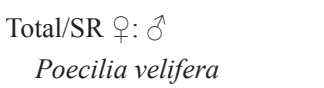 & - & - & $22.88 * / 1.85$ & $25.46 * / 1.69$ & - \\
\hline January-February & - & - & 2.00 & - & - \\
\hline March-April & - & - & 0.00 & - & - \\
\hline May-June & - & - & 0.00 & - & - \\
\hline July-August & - & - & $11.58^{*}$ & - & - \\
\hline September-October & - & - & $4.90^{*}$ & - & - \\
\hline
\end{tabular}


Tab. 3. (continued)

\begin{tabular}{|c|c|c|c|c|c|}
\hline Species/Sex ratio & Boa Vista & Pinheiros & Santo Antônio & Chato & Gavião \\
\hline November-December & - & - & 0.66 & - & - \\
\hline Total/SR $+:{ }^{\pi}$ & - & - & $12.90 * / 1.83$ & - & - \\
\hline \multicolumn{6}{|l|}{ Xiphophorus hellerii } \\
\hline January-February & 2.46 & 3.76 & $10.66^{*}$ & $10.34 *$ & $47.12 *$ \\
\hline March-April & 2.66 & $9.32 *$ & 1.00 & $28.60 *$ & $89.72 *$ \\
\hline May-June & $28.58^{*}$ & 0.80 & $13.50 *$ & $8.68 *$ & $35.28 *$ \\
\hline July-August & $11.36^{*}$ & $14.28 *$ & 1.00 & $7.18 *$ & $66.22 *$ \\
\hline September-October & 0.42 & $22.50^{*}$ & $5.06^{*}$ & 2.78 & $69.20^{*}$ \\
\hline November-December & 0.50 & $11.76^{*}$ & $5.36^{*}$ & 0.02 & $45.74 *$ \\
\hline Total/SR $+:{ }^{\lambda}$ & $36.00 * / 2.5$ & $55.34 * / 3.40$ & $31.64 * / 1.93$ & $50.58 * / 1.86$ & $345.75 * / 7.05$ \\
\hline \multicolumn{6}{|l|}{ Xiphophorus maculatus } \\
\hline January-February & 0.88 & 0.66 & 2.88 & 0.14 & 3.62 \\
\hline March-April & 0.34 & $12.24 *$ & 0.00 & $22.74 *$ & $28.34 *$ \\
\hline May-June & 0.58 & $7.12 *$ & 1.80 & 1.04 & $5.26^{*}$ \\
\hline July-August & 0.03 & $3.86^{*}$ & 0.52 & 0.36 & 3.00 \\
\hline September-October & 0.22 & 0.68 & 0.00 & $6.12 *$ & 0.74 \\
\hline November-December & 1.34 & $15.52^{*}$ & 0.02 & 0.02 & 3.04 \\
\hline Total/SR $+:{ }^{\pi}$ & $0.00 / 1$ & $24.20 * / 1.90$ & $0.06 / 0.97$ & $26.44 * / 1.67$ & $38.98 * / 1.71$ \\
\hline \multicolumn{6}{|l|}{ Xiphophorus variatus } \\
\hline January-February & - & 0.00 & 1.00 & $5.46^{*}$ & $41.68^{*}$ \\
\hline March-April & - & 0.06 & $4.00 *$ & $32.58^{*}$ & $4.64 *$ \\
\hline May-June & - & 0.00 & 0.34 & $49.00 *$ & $11.52 *$ \\
\hline July-August & - & 0.56 & $50.76^{*}$ & $34.10^{*}$ & $7.90^{*}$ \\
\hline September-October & - & 2.36 & $58.78^{*}$ & $25.00^{*}$ & $16.46^{*}$ \\
\hline November-December & - & 0.52 & $10.66^{*}$ & $11.92 *$ & $26.42 *$ \\
\hline Total/SR $+:{ }_{+}$ & - & $1.16 / 1.15$ & $116.74 * / 3.71$ & $83.50 * / 2.73$ & $92.82 * / 2.03$ \\
\hline
\end{tabular}

Tab. 4. Spearman's rank correlation between ornamental non-native reproductive female poeciliids and abiotic variables in the creeks in 2003, 2004, 2005 and 2006.

\begin{tabular}{|c|c|c|c|c|c|c|c|c|c|c|}
\hline \multirow{2}{*}{ Species/Variables } & \multicolumn{2}{|c|}{ Boa Vista } & \multicolumn{2}{|c|}{ Pinheiros } & \multicolumn{2}{|c|}{ Santo Antônio } & \multicolumn{2}{|c|}{ Chato } & \multicolumn{2}{|c|}{ Gavião } \\
\hline & $\mathrm{r}$ & $\mathrm{P}$ & $\mathrm{r}$ & $\mathrm{P}$ & $\mathrm{r}$ & $\mathrm{P}$ & $\mathrm{r}$ & $\mathrm{P}$ & $\mathrm{r}$ & $\mathrm{P}$ \\
\hline \multicolumn{11}{|l|}{ Poecilia reticulata } \\
\hline Gravid females $\times$ Water temperature & -0.883 & 0.07 & 0.116 & 0.83 & -0.522 & 0.29 & 0.580 & 0.23 & -0.551 & 0.26 \\
\hline Gravid females $\times$ Water level & -0.143 & 0.79 & 0.200 & 0.70 & -0.600 & 0.21 & 0.371 & 0.47 & -0.543 & 0.27 \\
\hline Gravid females $\times$ Rainfall & -0.257 & 0.62 & 0.200 & 0.70 & -0.543 & 0.27 & 0.428 & 0.40 & -0.428 & 0.40 \\
\hline \multicolumn{11}{|l|}{ Poecilia sphenops } \\
\hline Gravid females $\times$ Water temperature & - & - & - & - & -0.232 & 0.66 & 0.116 & 0.83 & - & - \\
\hline Gravid females $\times$ Water level & - & - & - & - & 0.143 & 0.79 & -0.086 & 0.87 & - & - \\
\hline Gravid females $\times$ Rainfall & - & - & - & - & -0.086 & 0.87 & -0.028 & 0.96 & - & - \\
\hline \multicolumn{11}{|l|}{ Poecilia velifera } \\
\hline Gravid females $\times$ Water temperature & - & - & - & - & 0.279 & 0.59 & - & - & - & - \\
\hline Gravid females $\times$ Water level & - & - & - & - & 0.464 & 0.35 & - & - & - & - \\
\hline Gravid females $\times$ Rainfall & - & - & - & - & 0.406 & 0.42 & - & - & - & - \\
\hline \multicolumn{11}{|l|}{ Xiphophorus hellerii } \\
\hline Gravid females $\times$ Water temperature & 0.530 & 0.28 & -0.058 & 0.91 & -0.116 & 0.83 & 0.580 & 0.23 & 0.783 & 0.07 \\
\hline Gravid females $\times$ Water level & 0.771 & 0.07 & 0.314 & 0.54 & 0.257 & 0.62 & 0.428 & 0.40 & 0.600 & 0.21 \\
\hline Gravid females $\times$ Rainfall & 0.829 & 0.06 & 0.314 & 0.54 & -0.028 & 0.96 & 0.543 & 0.27 & 0.771 & 0.07 \\
\hline \multicolumn{11}{|l|}{ Xiphophorus maculatus } \\
\hline Gravid females $\times$ Water temperature & 0.765 & 0.08 & -0.145 & 0.78 & 0.015 & 0.98 & 0.754 & 0.08 & 0.725 & 0.10 \\
\hline Gravid females $\times$ Water level & 0.486 & 0.33 & -0.314 & 0.54 & 0.087 & 0.87 & 0.486 & 0.33 & 0.314 & 0.54 \\
\hline Gravid females $\times$ Rainfall & 0.543 & 0.27 & -0.314 & 0.54 & 0 & 1 & 0.657 & 0.16 & 0.600 & 0.60 \\
\hline \multicolumn{11}{|l|}{ Xiphophorus variatus } \\
\hline Gravid females $\times$ Water temperature & - & - & -0.696 & 0.12 & 0.031 & 0.21 & -0.029 & 0.96 & -0.029 & 0.96 \\
\hline Gravid females $\times$ Water level & - & - & -0.257 & 0.62 & 0.698 & 0.12 & -0.200 & 0.70 & 0.486 & 0.33 \\
\hline Gravid females $\times$ Rainfall & - & - & -0.257 & 0.62 & 0.577 & 0.23 & -0.028 & 0.57 & 0.314 & 0.54 \\
\hline
\end{tabular}




\section{Discussion}

Population studies of non-native poeciliid species are rare in Brazil and this study provides a unique opportunity to evaluate the establishment of 22 populations of six ornamental non-native poeciliids in a same geographic region. We found females of $P$. reticulata, $P$. sphenops, $X$. hellerii, $X$. maculatus, and $X$. variatus in reproductive activity during the 12 months of the year in almost all creeks, with the exception of the guppy in Boa Vista and Yucatan molly in Santo Antônio creeks, that have reproductive periods of 10 and eight months respectively. Frequent reproduction over an extended breeding season is an opportunistic life-history strategy to overcome hydrologically unstable environments such as creeks and streams that are subject to flash floods, and provides a mechanism to maintain and/or reestablish the structure of population in poeciliid fishes (Winemiller, Rose, 1992). In this way, the continuous input of newborn individuals would assure a higher survival rate and therefore the maintenance of a viable population in a stochastic environment (Abilhoa et al., 2011). We also found gravid females of all non-native species with small sizes, which can indicate probable dwarfing or stunting. This phenomenon may be due to three reasons in the studied creeks: (i) intraspecific and interspecific competition due to overcrowding, (ii) low autochthonous food resources availability (typical of headwater habitats) and; (iii) increased survival rate due to the absence of large top predators (Olinger et al., 2016).

Juveniles of all six species were caught in all sites. The presence of juveniles is an indication of successful recruitment, and suggests that all 22 populations are established in these headwater creeks, which provide similar conditions to those of their places of origin, in tropical regions of Central and South America (Eschmeyer et al., 2016). All six poeciliid species produced small broods in comparison with larger broods recorded for these species introduced in other countries (Sa-nguansil, Lheknim, 2010; Froese, Pauly, 2016). According to Olinger et al. (2016), in upstream habitats (i.e. headwaters), female poeciliids have been shown to exhibit small broods and larger fingerlings when resources are scarce. Small broods may give these non-native livebearers a competitive advantage over native oviparous species, because fry are larger, feed at birth, grow more quickly and become predators' faster (Rupp, 1996; Olinger et al., 2016).

A higher frequency of females (female-biased sex ratios), as was found in all creeks, appears to be common in other tropical regions. In poeciliids, males are more susceptible to mortality from a variety of sources, including differential predation (due to their bright colours), higher susceptibility to stressors, such as extreme temperatures, overcrowding, hypoxia, and also accelerated aging, since they invest their energy exclusively in mating behaviors (Snelson, 1989). Moreover, females do not depend on the constant presence of males to reproduce because they are able to retain active sperm in ovarian tissues for long periods and to bear successive broods without reimpregnation. These attributes of invasiveness allow them to colonize any environment by themselves (Deacon et al., 2011). The lack of correlation between abiotic factors was already expected, particularly considering the favourable temperature conditions throughout the years. This has been explained by ovoviviparity, a common reproductive strategy of the studied species (Snelson, 1989). Since eggs and embryos develop inside the female's body, they are less influenced by several external conditions such as temperature variations, rain, diseases and predators attack (Oliveira et al., 2014).

The presence of reproductive adults and juveniles of non-native poeciliids in these creeks, besides characterizing establishment by recruitment, may lead to biotic homogenization, i.e. the establishment of the same non-native species at two or more locations, decreasing beta-diversity over time (Olden et al., 2011). This situation is already occurring in the area (Magalhães et al., 2011), as well as the probable intra-specific hybridization between $X$. maculatus and X. variatus (A.L.B. Magalhães, pers. obs.). Likewise, it is also feasible that non-native Poecilia species hybridize with native congeners (e.g. P. vivipara), increasing the risk of native species extinction. According to Meyer et al. (2006) and Lampert, Schartl (2008), hybridization within Xiphophorus Heckel 1848 and Poecilia Bloch \& Schneider 1801 is fairly common. Inter-specific competition for shelter, food and parturition sites could arise between non-native poeciliids and the livebearer endemic genus Phalloceros Eigenmann 1907 (Menezes et al., 2007). The nesting-guarding cichlids such as the chameleon cichlid Australoheros muriae Ottoni \& Costa 2008, pearl cichlid Geophagus obscurus (Castelnau 1855) (= Geophagus brasiliensis Quoy \& Gaimard 1824) and the loricariids Hypostomus affinis (Steindachner 1877) and Neoplecostomus microps (Steindachner 1877) will probably be unaffected by livebearers, but other native species that do not guard their eggs (e.g. lambari Astyanax janeiroensis Eigenmann 1908, lambari Astyanax giton Eigenmann 1908, catfishes Trichomycterus spp.) (Magalhães, Jacobi, 2013a), could be adversely affected. Species loss due to the influence of non-native poeciliids could be devastating because, according to Cruz et al. (2013), in systems with few native fish species such as headwater creeks, the loss of a single species might represent the deletion of an entire component of the food chain, compromising ecosystem functioning.

Although springheads and headwater creeks are legally considered permanent preservation areas they are often overlooked by conservation efforts in Brazil (Callisto et al., 2012). Given the current and future adverse ecological impacts derived from the presence of non-native poeciliids in these ecosystems, we propose management recommendations grouped into three categories: prevention, remediation and research. Preventing incursions of organisms outside of their native ranges is the most cost-effective way 
and public policies are needed to protect Brazil's headwater creeks from future introductions of new stocks of poeciliids and other non-native species. Ornamental aquaculture, as the main cause for poeciliid presence and spread throughout Brazil (Bizerril, Lima, 2001; Castellani, Barrella, 2006; Alves et al., 2007; Magalhães, Jacobi, 2008), should be controlled more carefully. We echo recommendations of Courtenay et al. (1974) in rerouting all effluent waters from fish rearing facilities through a dry well, and installation of adequate sand and gravel filter to allow passage of water but not livestock. We also suggest periodic inspection of ornamental fish farms to confirm the application of these measures. In addition, the Polluter Pays Principle should be enforced, since the introduction of non-native species is regarded as a form of environmental pollution, and environmental awareness campaigns on the negative effects of non-native species release into the wild should also be used, as proposed before (Magalhães, Jacobi, 2008).

Control or eradication with rotenone to remove nonnative species that can compete with native fishes, selective removal of non-native species using electrofishing and netting, elimination of non-native grasses at the margins may remove protection/feeding sites and reduce abundance of vegetation-dependent forms (i.e. non-native juveniles), native species reintroduction to the reclaimed habitats followed by habitat restoration activities such as flow restoration, introduction of woody debris or riparian vegetation rehabilitation (Kennard et al., 2005; Rayner, Creese, 2006; Casatti et al., 2009) are all measures that will only be effective if the prevention policies are put into practice.

Studies about direct effects of non-native poeciliids on native competitor species are needed, with special emphasis on hybridization and adult competition for shelter, food, and reproductive sites (Alves et al., 2007). When non-native poeciliids of the genera Poecilia and Xiphophorus co-occur, negative impacts are observed on native communities of small water bodies (Arthington et al., 1983; Englund, 1999; Goren, Galil, 2005; Stockwell, Henkanaththegedara, 2011), and this situation already occurs in a headwater creek located in southern Brazil (Vieira, Shibatta, 2007). The use of artificial refuges could also promote coexistence between non-native poeciliids and native species (Westhoff et al., 2013; Magellan, García-Berthou, 2016).

Finally, careful management of poeciliid introductions can help Brazil avoid the catastrophic invasions that have occurred elsewhere (Arthington et al., 1983; Englund, 1999; Goren, Galil, 2005; Stockwell, Henkanaththegedara, 2011). The establishment of non-native poeciliids in Brazilian headwater creeks is the successful combination of appeal to humans (popular and inexpensive aquarium fish), propagule pressure (multiple introduction events), invasiveness (extended breeding season, small broods of large embryos, female-biased sex ratio, sperm storage, independence of external environmental conditions to reproduce), and invasibility (tropical region, low native species richness, absence of top predators). These fish already have and will continue to have significant impacts in the native biological communities of headwater creek ecosystems if the recommendations above, in particular those concerning cessation of propagule pressure, are not implemented.

\section{Acknowledgments}

We thank the many ornamental fish farmers who granted access to their properties, and in particular farmer Jorge Farias for field assistance, Roberto E. Reis (MCPPUCRS) and Renata B. Araújo (CIUFS-UFS) for the species catalogue numbers, the Programa de Pós-Graduação em Ecologia, Conservação e Manejo de Vida Silvestre (Universidade Federal de Minas Gerais) and the US Fish and Wildlife Service for financial support. ALBM received a research grant of Coordenação de Aperfeiçoamento de Pessoal de Nível Superior (CAPES) and CMJ thanks the Conselho Nacional de Desenvolvimento Científico e Tecnológico $(\mathrm{CNPq})$ for a Research Productivity fellowship. We also thank two anonymous reviewers for their valuable comments.

\section{References}

Abell R, Allan JD, Lehner B. Unlocking the potential of protected areas for freshwaters. Biol Cons. 2007; 134(1):48-63.

Abilhoa V, Braga RR, Bornatowski H, Vitule JRS. Fishes of the Atlantic Rain Forest streams: ecological patterns and conservation. In: Grillo O, Venora G, editors. Changing diversity in changing environment. Rijeka: InTech; 2011. p.259-82.

Albert JS, Reis RE. Historical biogeography of Neotropical freshwater fishes. Los Angeles: University of California Press; 2011.

Alves CBM, Vieira F, Magalhães ALB, Brito MFG. Impacts of nonnative fish species in Minas Gerais, Brazil: present situation and prospects. In: Bert TM, editor. Ecological and genetic implications of aquaculture activities. Dordrecht: Springer Press; 2007. p.291-314.

Andrade PM, Braga FMS. Reproductive seasonality of fishes from a lotic stretch of the Grande River, high Paraná river basin, Brazil. Braz J Biol. 2005; 65(3):387-94.

Arthington AH, Milton DA, McKay RJ. Effects of urban development and habitat alterations on the distribution and abundance of native and exotic freshwater fish in the Brisbane region, Queensland. Austral Ecol. 1983; 8(2):87-101.

Assis DAS, Cavalcante SS, Brito MFG. Avaliação do comércio de peixes ornamentais de água doce em Aracaju, Sergipe. Magistra. 2014; 26(2):213-20.

Bizerril CRSF, Lima NRW. Espécies de peixes introduzidos nos ecossistemas aquáticos continentais do estado do Rio de Janeiro, Brasil. Comun Mus Cienc Tecnol PUCRS Ser. Zool. 2001; 14(1):43-59. 
Callisto M, Melo AS, Baptista DF, Gonçalves Júnior JF, Graça MAF, Augusto FG. Future ecological studies of Brazilian headwater streams under global-changes. Acta Limnol Bras. 2012; 24(3):293-302.

Cardoso RS, Igarashi MA. Aspectos do agronegócio da produção de peixes ornamentais no Brasil e no mundo. Pubvet. 2009; 3(15):1-22.

Cardoso RS, Lana AMQ, Teixeira EA, Luz RK, Faria PMC. Caracterização socioeconômica da aquicultura ornamental na região da Zona da Mata Mineira. Bol Inst Pesca. 2012; 38(1):89-96.

Casatti L, Ferreira CP, Carvalho FR. Grass-dominated stream sites exhibit low fish species diversity and dominance by guppies: an assessment of two tropical pasture river basins. Hydrobiologia. 2009; 632(1):273-83.

Castellani D, Barrella W. Impactos da atividade de piscicultura na bacia do Rio Ribeira do Iguape, SP - Brasil. Bol Inst Pesca. 2006; 32(2):161-71.

Coe CM, Freitas MC, Araújo RCP. Diagnóstico da cadeia produtiva de peixes ornamentais no município de Fortaleza, Ceará. Magistra. 2011; 23(3):107-14.

Courtenay Jr. WR, Sahlman HF, Miley II WW, Herrema DJ. Exotic fishes in fresh and brackish waters of Florida. Biol Cons. 1974; 6(4):292-302.

Cruz BB, Teshima FA, Cetra M. Trophic organization and fish assemblage structure as disturbance indicators in headwater streams of lower Sorocaba River basin, São Paulo, Brazil. Neotrop Ichthyol. 2013; 11(1):171-78.

Cuvier G, Valenciennes A. Histoire naturelle des poissons. Tome dix-huitième. Suite du livre dix-huitième. Paris: Libraire de la Société Géologique de France; 1846.

Deacon AE, Ramnarine IW, Magurran AE. How reproductive ecology contributes to the spread of a globally invasive fish. PLoS One. 2011; 6(9): e24416.

Dudgeon D, Arthington AH, Gessner MO, Kawabata Z-I, Knowler DJ, Lévêque C, Naiman RJ, Prieur-Richard A-H, Soto D, Stiassny MLJ, Sullivan CA. Freshwater biodiversity: importance, threats, status and conservation challenges. Biol Rev Camb Philos. 2006; 81(2):163-82.

Englund RA. The impacts of introduced poeciliid fish and Odonata on the endemic Megalagrion (Odonata) damselflies of Oahu Island, Hawaii. J Insect Conserv. 1999; 3(3):225-43.

Eschmeyer WN, Fricke R, van der Laan R, editors. Catalog of fishes: genera, species, references. [Electronic Version]. San Francisco (CA): California Academy of Sciences; 2016. [cited 2016 Jun 19]. Available from: http:// researcharchive.calacademy.org/research/ichthyology/ catalog/fishcatmain.asp

Froese R, Pauly D, editors. FishBase. [World Wide Web electronic publication]. Penang (MA), Rome: FAO; 2016 [cited 2016 Sep 06]. Available from: http://fishbase.org

Garcia DAZ, Costa ADA, Yabu MHS, Balconi APR, Orsi ML. Sobre como peixes de outras bacias chegam às nossas águas: o caso do rio Paranapanema, bacia do alto Paraná. Bol Soc Bras Ictiol. 2014; (110):8-15.
Gkenas C, Oikonomou A, Economou A, Kiosse F, Leonardos I. Life history pattern and feeding habits of the invasive mosquitofish, Gambusia holbrooki, in Lake Pamvotis (NW Greece). J Biol Res-Thessalon. 2012; (17):121-36.

Goren M, Galil BS. A review of changes in the fish assemblages of Levantine inland and marine ecosystems following the introduction of non-native fishes. J Appl Ichthyol. 2005; 21(4):364-70.

Günther A. Catalogue of the Physostomi, containing the families Salmonidae, Percopsidae, Galaxidae, Mormyridae, Gymnarchidae, Esocidae, Umbridae, Scombresocidae, Cyprinodontidae, in the collection of the British Museum. London:British Museum; 1866. (Catalogue of fishes in the British Museum; vol 6).

Hammer Ø, Harper DAT, Ryan PD. Past - Paleontological statistics software package for education and data analysis. Version 1.91[Internet]. 2009. Available from: http://folk. uio.no/ohammer/past

Haynes JL. Standardized classification of poeciliid development for life-history studies. Copeia. 1995; 1995(1):147-54.

Heckel JJ. Eine neue gattung von Poecilien mit rochenartigem anklammerungs-organe. Sitzungsber Kaiser Akad Wiss

Math-Naturwiss K1. 1848; 1: 289-303.

Institutional Animal Care and Use Committee (IACUC). Institutional Animal Care and Use Committee Guidebook. 2nd ed. Washington DC: Department of Health and Human Services; 2002.

Instituto Nacional de Pesquisas Espaciais (INPE). Dados observacionais; 2006 [cited 2006 Dec 31]. Available from: http://www.cptec.inpe.br/dados_observados/

Kennard MJ, Arthington AH, Pusey BJ, Harch BD. Are alien fish a reliable indicator of river health? Freshwater Biol. 2005; 50(1):174-93.

Lampert KP, Schartl M. The origin and evolution of a unisexual hybrid: Poecilia formosa. Philos Trans R Soc, B. 2008; 363(1505):2901-09.

Le Sueur CA. Description of a new genus, and of several new species of fresh water fish, indigenous to the United States. Proc Acad Nat Sci Philadelphia. 1821; 2(pt 1):2-8.

Lucinda PHF. Systematics and biogeography of the genus Phalloceros Eigenmann, 1907 (Cyprinodontiformes: Poeciliidae: Poeciliinae), with the description of twentyone new species. Neotrop Ichthyol. 2008; 6(2):113-58.

Magalhães ALB, Casatti L, Vitule JRS. Alterações no Código Florestal Brasileiro favorecerão espécies não-nativas de peixes de água doce. Nat Conserv. 2011; 9(1):121-24.

Magalhães ALB, Jacobi CM. Ornamental exotic fish introduced into Atlantic Forest water bodies, Brazil. Neotrop Biol Conserv. 2008; 3(2):73-77.

Magalhães ALB, Jacobi CM. Asian aquarium fishes in a Neotropical biodiversity hotspot: impeding establishment, spread and impacts. Biol Invasions. 2013a; 15(10):2157-63.

Magalhães ALB, Jacobi CM. Invasion risks posed by ornamental freshwater fish trade to southeastern Brazilian rivers. Neotrop Ichthyol. 2013b; 11(2):433-41. 
Magellan K, García-Berthou E. Experimental evidence for the use of artificial refugia to mitigate the impacts of invasive Gambusia holbrooki on an endangered fish. Biol Invasions. 2016; 18(3):873-82.

Meek SE. The fresh-water fishes of Mexico north of the isthmus of Tehuantepec. Chicago: Field Columbian Museum; 1904. (Field Columbian Museum. Publication, Zoological series; vol 5).

Menezes NA, Weitzman SH, Oyakawa OT, Lima FCT, Castro RMC, Weitzman MJ. Freshwater fishes of Mata Atlântica: preliminary list of species and comments on conservation of Neotropical freshwater fishes. São Paulo: Editora do Museu de Zoologia; 2007.

Meyer A, Salzburger W, Schartl M. Hybrid origin of a swordtail species (Teleostei: Xiphophorus clemenciae) driven by sexual selection. Mol Ecol. 2006; 15(3):721-30.

Milton DA, Arthington AH. Reproductive biology of Gambusia affinis holbrooki Baird and Girard, Xiphophorus hellerii (Günther) and X. maculatus (Heckel) (Pisces, Poeciliidae) in Queensland, Australia. J Fish Biol. 1983; 23(1):23-41.

Olden JD, Lockwood JL, Parr CL. Biological invasions and the homogenization of faunas and floras. In: Ladle RJ, Whittaker RJ, editors. Conservation biogeography. Oxford: Blackwell Publishing Ltd; 2011. p.224-243.

Olinger CT, Peoples BK, Frimpong EA. Reproductive life history of Heterandria bimaculata (Heckel, 1848) (Poeciliinae: Poeciliidae) in the Honduran interior highlands: trait variation along an elevational gradient. Neotrop Ichthyol. [serial on the Internet]. 2016; 14(1):e150050. Available from: http://dx.doi. org/10.1590/1982-0224-20150050

Oliveira TD, Reis AC, Guedes CO, Sales ML, Braga EPR, Ratton TF, Maia BP, Magalhães ALB. Establishment of non-native guppy Poecilia reticulata (Peters, 1859) (Cyprinodontiformes: Poeciliidae) in an Municipal Park located in Minas Gerais State, Brazil. PANAMJAS. 2014; 9(1):21-30.

Peters W. Eine neue vom Herrn Jagor im atlantischen Meere gefangene Art der Gattung Leptocephalus, und über einige andere neue Fische des Zoologischen Museums. Monatsberichte der Königlichen Preussischen Akademie der Wissenschaften zu Berlin. 1859; 1859: 411-13.

Pope KL, Lochmann SE, Young MK. Methods for assessing fish populations. In: Quist MC, Hubert WA, editors. Inland fisheries management in North America. 3rd edition. Bethesda: American Fisheries Society; 2010. p.325-351.

Rayner TS, Creese RG. A review of rotenone use for the control of non-indigenous fish in Australian fresh waters, and an attempted eradication of the noxious fish, Phalloceros caudimaculatus. New Zeal J Mar Fresh. 2006; 40(3):477-86.

Regan CT. Description of a new cyprinodont fish of the genus Mollienisia from Yucatan. Ann Mag nat Hist (series 8). 1914; 13(75):338.
Rupp HR. Adverse assessments of Gambusia affinis: an alternative view for mosquito control practitioners. J Am Mosq Control Assoc. 1996; 12(2):155-59.

Sa-nguansil S, Lheknim V. The occurrence and reproductive status of Yucatan molly Poecilia velifera (Regan, 1914) (Poeciliidae; Cyprinodontiformes): an alien fish invading the Songkhla Lake Basin, Thailand. Aquat Invasions. 2010; 5(4):423-30.

Sidle RC, Tsuboyama Y, Noguchi S, Hosoda I, Fujieda M, Shimizu T. Stormflow generation in steep forested headwaters: a linked hydrogeomorphic paradigm. Hydrol Process. 2000; 14(3):369-85.

Snelson Jr. FF. Social and environmental control of life history traits in poeciliid fishes. In: Snelson Jr. FF, Meffe GK, editors. Ecology and evolution of livebearing fishes. New Jersey: Prentice Hall; 1989. p.149-161.

Sokal RR, Rohlf FJ. Biometry: the principles and practice of statistics in biological research. 3rd edition. New York: W.H. Freeman; 1995.

Stockwell CA, Henkanaththegedara SM. Evolutionary conservation biology. In: Evans JP, Pilastro A, Schlupp I, editors. Ecology and evolution of Poeciliid fishes. Chicago: University of Chicago Press; 2011. p.128-141.

Strahler AN. Quantitative analysis of watershed geomorphology. Eos Trans AGU. 1957; 38(6):913-20.

Vieira DB, Shibatta OA. Peixes como indicadores de qualidade ambiental do ribeirão Esperança, município de Londrina, Paraná, Brasil. Biota Neotrop. 2007; 7(1):1-9.

Vieira F, Rodrigues RR. A fauna de peixes dos afluentes do rio Paraíba do Sul no estado de Minas Gerais. MG.BIOTA. 2010; 3(1):5-23.

Westhoff JT, Watts AV, Mattingly HT. Efficacy of artificial refuge to enhance survival of young Barrens topminnows exposed to western mosquitofish. Aquat Conserv. 2013; 23(1):65-76.

Williamson M. Variation in the rate and pattern of spread in introduced species and its implications. In: Perrings C, Mooney $\mathrm{H}$, Williamson $\mathrm{M}$, editors. Bioinvasions and Globalization: ecology, economics, management and policy. Oxford: Oxford University Press; 2000. p.56-65.

Winemiller KO. Seasonality of reproduction by livebearing fishes in tropical rainforest streams. Oecologia. 1993; 95(2):266-76.

Winemiller KO, Rose KA. Patterns of life-history diversification in North American fishes: implications for population regulation. Can J Fish Aquat Sci. 1992; 49(10):2196-218.

Submitted June 21, 2016 Accepted October 30, 2016 by Katya Kovalenko 
